\title{
Current status of assisted reproductive technology in Korea, 2010
}

\author{
Gyoung Hoon Lee', Hyun Jin Song' ', Kyu Sup Lee' ${ }^{2}$ Young Min Choi, \\ 'I-one Center, Seoul Women's Hospital, Bucheon; 2Department of Obstetrics and Gynecology, Pusan National University School of Medicine and \\ Medical Research Institute, Pusan National University Hospital, Busan; ${ }^{3}$ Department of Obstetrics and Gynecology, Seoul National University College of \\ Medicine, Seoul; ${ }^{4}$ The Institute of Reproductive Medicine and Population, Medical Research Center, Seoul National University College of Medicine, \\ Seoul, Korea
}

Objective: Great advances have been made in the field of assisted reproductive technology (ART) since the first in vitro fertilization (IVF) baby was born in Korea. This study was designed to report on the current status of ART therapy in South Korea between January 1 and December 31 of 2010.

Methods: A revised survey, originally developed by the International Committee Monitoring Assisted Reproductive Technologies, was sent to all available ART centers via email in 2013. Fresh embryo transfer (FET) cases were categorized into standard IVF or intracytoplasmic sperm injections. These cases, the thawing embryo transfer (TET) cases, and other related procedures were surveyed.

Results: Data from 30,785 ART procedures were provided by 78 clinics. Of the 28,200 cycles in which oocytes were retrieved, $92.2 \%$ of these cycles were completely transferred. In addition, 8,075 cycles were confirmed to be clinical pregnancies in the FET cycles, which represent a pregnancy rate of $28.6 \%$ per oocyte pick-up and $31.1 \%$ per embryo transfer. The most common number of embryos transferred in the FET was three embryos (37.3\%) followed by two embryos (36.3\%) and one embryo (14.0\%). Of the 6,648 TET cycles transferred, 2,356 clinical pregnancies were confirmed by ultrasonography. The most common number of embryos in the TET group was two embryos (43.4\%) followed by three embryos (25.4\%) and one embryo (18.9\%).

Conclusion: The clinical pregnancy rate per transfer in the FET cycles was similar in 2009 and 2010. Among the FET cycles where one or two embryos were transferred, the clinical pregnancy rate per transfer slightly increased from 2009 (28.7\%) to 2010 (32.9\%).

Keywords: Assisted reproductive technology; International Committee for Monitoring Assisted Reproductive Technologies; Korea; Survey

\section{Introduction}

Ever since the first in vitro fertilization (IVF) baby was born in South Korea (hereafter Korea) in 1985, use of assisted reproductive technology (ART) to overcome infertility has increased sharply with the simultaneous increase in the number of the fertility centers.

Received: Dec 9, 2014 · Revised: Jan 11, 2015 · Accepted: Feb 24, 2015 Corresponding author: Young Min Choi

Department of Obstetricsand Gynecology, The Institute of Reproductive Medicine and Population, Medical Research Center, Seoul National University College of Medicine, 103 Daehak-ro, Jongno-gu, Seoul 110-799, Korea Tel:+82-2-2072-2385 Fax:+82-2-762-3599 E-mail:ymchoi@snu.ac.kr

This is an Open Access article distributed under the terms of the Creative Commons Attribution Non-Commercial License (http://creativecommons.org/licenses/by-nc/3.0/) which permits unrestricted non-commercial use, distribution, and reproduction in any medium, provided the original work is properly cited.
In 1992, the Korean Society of Obstetrics and Gynecology published their first report. Since then, the Korean Society of Assisted Reproduction (KSAR) has revised the data field queries in the voluntary registration of ART data. Two other evaluation programs exist in Korea. Of these, the National Supporting Program for Infertility was implemented in 2006. Facing the risk of low birth rates and high financial burdens associated with the ART procedures, the Korean government implemented a financial support program for infertile couples who fall below a certain annual income, and then these outcomes are reported annually. Thus, the quality of the data reported by the National Supporting Program for Infertility is assured. The second evaluation program is organized by the National Assembly of Korea. In 2005, they passed the Bioethics and Safety Act that requires all the fertility centers in Korea to provide an annual report on the status of 
embryos generated for treatment and/or research purposes to the Korean Ministry of Health and Welfare through a validation process, which is authorized by public officials.

The data on these ART procedures from fertility centers has been considered an abundant, valuable resource. Furthermore, the direction of the use of fertility treatments in Korea has been speculated based on long-term analyses of the ART data.

The purpose of this survey was to gather data on the status and success rate of each ART clinic, which provided voluntary data on their procedures performed in Korea between January and December of 2010.

\section{Methods}

\section{Subjects}

Among the one-hundred and nineteen ART clinics that had received registration forms amended by the Committee of Statistics in the KSAR, seventy-six clinics reported their outcomes (return rate $=68.1 \%$ ). The complete list of the ART clinics that participated in this survey is listed by region in Supplement 1.

\section{Methods}

A questionnaire survey was submitted in Korean language to all of the ART clinics. Responses were collected via e-mail. The survey was originally based on the International Committee Monitoring Assisted Reproductive Technologies Tool Box for Data Collection forms at the national and regional levels; however, the the Committee of Statistics, KSAR revised the questionnaire survey at 2013 (Supplement 2).

In the current questionnaire survey, the IVF data were categorized into outcomes (1) after embryo transfer (ET) at fresh cycles or (2) after frozen ET according to the IVF methods, which include conventional IVF and intracytoplasmic sperm injection (ICSI) as well as other related procedures.

All data are related to the treatment cycles in 2010. However, the data on the outcomes oocytes pick-up or controlled ovarian stimulation per day was somewhat arbitrarily reported by each clinic; thus, a mixed dataset was created. Clinical pregnancy was defined only in the cases that presented with an intrauterine gestational sac on ultrasound examination. Cases of biochemical pregnancy were excluded if only serum beta human chorionic gonadotropin was elevated. Live birth delivery was defined as a birth of one or more live born infants, with delivery of multiple infants counting as one live-birth delivery.

\section{Analysis}

The contribution of ART to each outcome was calculated by dividing the total number of outcomes by the number of oocytes pick-up and number of ETs. All of the data analyses were performed using
PASW ver. 18.0 (SPSS Inc., Chicago, IL, USA).

\section{Results}

\section{Overview}

1) Data provided by the fertility clinics

Seventy-eight centers reported the treatment cycles that had been initiated in 2010. In total, 30,785 cycles of ART treatment were reported, while the initiation criteria differed across the clinics. The initiation criteria was based on either the day ovarian stimulation was medically induced (11 clinics), the day oocytes were retrieved (42 clinics), or contained no comment on their initiation criterion (25 clinics). The number of ART cycles increased by $10.0 \%$ from 27,973 cycles (74 centers) in 2009 to 30,785 cycles (78 centers) in 2010.

\section{2) Type of ART procedures}

Among the 30,785 ART cycles, fresh ET were used in approximately $75 \%$ of these ART cycles performed in $2010(n=23,202)$. ART cycles that used frozen embryos were the next most common type of procedure, accounting for approximately $22.6 \%$ of the total number of cycles $(n=6,945)$. The less common procedures in 2010 included oocyte donation ( $n=213)$, preimplantation genetic diagnosis $(n=424)$, and gamete intrafallopian tube transfer $(n=1)$ (Table 1).

\section{3) Trends by age}

For the fresh ET cycle, women aged 35-39 represented the majority (38.0\%) of all fresh ET cycles performed in 2010. The age distribution among patients who received fresh ET cycles is as follows: $35.4 \%$ among those aged 30-34, 19.7\% among those aged over 40, 6.7\% among those aged 25-29, and $0.3 \%$ among those younger than 25 . For the thawing ET cycle, women aged 30-34 comprised the largest group of women, representing 54.5\%. Different from the age distribution in the fresh ET cycle, women aged 35-39 was the next most common age group. Of the total number of fresh ET cycles $(n=4,788)$ and thawing ET cycles $(n=660), 15.6 \%$ and $9.5 \%$ of these cycles, respectively, were missing data on age.

The majority of women who used a fresh ET cycle were aged 35-39 in 2010, whereas the majority of these women were aged 30-34 yr in 2009. The percentage of women aged over 40 who used a fresh ET

Table 1. The number of cycles for each ART procedure

\begin{tabular}{lcc}
\hline Type of ART procedure & No. of cycles in 2010 (\%) & No. of cycles in 2009 (\%) \\
\hline Fresh ET & $23,202(75.4)$ & $22,049(78.9)$ \\
Thawing ET & $6,945(22.6)$ & $5,704(20.4)$ \\
Others & $638(2.1)$ & $191(0.7)$ \\
Total & $30,785(100.0)$ & $27,944(100.0)$ \\
\hline
\end{tabular}

ART, assisted reproductive technology; ET, embryo transfer. 
cycle increased sharply from $11.9 \%$ in 2009 to $19.7 \%$ in 2010 . For the thawing ET cycle, the majority of women were aged 30-34 in 2010 (54.5\%) and 2009 (48.7\%) (Table 2).

\section{4) Clinical pregnancy by the patients' age}

Among patients who had retrieved oocytes ( $n=28,200), 92.2 \%$ of these patients ( $n=25,997)$ successfully transferred their embryos in 2010. Of these, 8,075 patients were confirmed to have achieved clinical pregnancy, which represents $28.6 \%$ oocytes retrieval per cycle and $31.1 \%$ ET per cycle. Among patients who had thawing ET $(n=6,922), 2,440$ patients were confirmed to have achieved clinical pregnancy, which represents a $35.2 \%$ clinical pregnancy rate per cycle with ET. Compared to the rate of fresh ET cycles, pregnancy rate for thawing ET cycles was higher among those aged 30-34 (Table 3).

\section{5) Live birth by treatment type}

Although many of the clinics that participated in this study may not have been able to follow-up with pregnant patients until the actual

Table 2. Trends of fresh ET and thawing ET by age group

\begin{tabular}{lccccc}
\hline \multirow{2}{*}{$\begin{array}{c}\text { Age group } \\
(\mathrm{yr})\end{array}$} & \multicolumn{2}{c}{ Fresh ET } & & \multicolumn{2}{c}{ Thawing ET } \\
\cline { 2 - 3 } \cline { 5 - 6 } & $2010(\%)$ & $2009(\%)$ & & $2010(\%)$ & $2009(\%)$ \\
\hline$<25$ & $70(0.3)$ & $320(1.0)$ & & $28(0.4)$ & $43(0.8)$ \\
$25-29$ & $1,738(6.7)$ & $4,878(14.9)$ & & $701(11.2)$ & $670(11.9)$ \\
$30-34$ & $9,192(35.4)$ & $13,781(42.0)$ & & $3,427(54.5)$ & $2,751(48.7)$ \\
$35-39$ & $9,883(38.0)$ & $9,923(30.3)$ & & $2,226(35.4)$ & $1,770(31.4)$ \\
$\geq 40$ & $5,114(19.7)$ & $3,901(11.9)$ & & $540(8.6)$ & $411(7.3)$ \\
Total & $25,997^{\text {a) }}(100.0)$ & $32,803(100.0)$ & & $6,285^{\text {a) }}(100.0)$ & $5,645(100.0)$ \\
\hline
\end{tabular}

ET, embryo transfer.

a)Patient age was missing in 4,788 fresh ET patients and 660 thawing ET patients.

Table 3. Clinical pregnancy by age group

\begin{tabular}{|c|c|c|c|c|c|c|c|c|c|c|c|c|}
\hline \multirow{2}{*}{ Outcome } & \multicolumn{6}{|c|}{ Fresh cycle } & \multicolumn{6}{|c|}{ Thawing cycle } \\
\hline & $\leq 24$ & $25-29$ & $30-34$ & $35-39$ & $\geq 40$ & All & $\leq 24$ & $25-29$ & $30-34$ & $35-39$ & $\geq 40$ & All \\
\hline No. of OPU & 76 & 1,930 & 9,933 & 10,561 & 5,700 & 28,200 & - & - & - & - & - & - \\
\hline ET cycles & 70 & 1,738 & 9,192 & 9,883 & 5,114 & 25,997 & 28 & 701 & 3,427 & 2,226 & 540 & 6,922 \\
\hline Clinical pregnancies & 28 & 749 & 3,518 & 3,026 & 754 & 8,075 & 16 & 249 & 1,284 & 760 & 131 & 2,440 \\
\hline CPR per OPU (\%) & 36.8 & 38.8 & 35.4 & 28.7 & 13.2 & 28.6 & - & - & - & - & - & - \\
\hline CPR per ET (\%) & 40.0 & 43.1 & 38.3 & 30.6 & 14.7 & 31.1 & 57.1 & 35.5 & 37.5 & 34.1 & 23.4 & 35.2 \\
\hline
\end{tabular}

OPU, oocyte pick-up; ET, embryo transfer; CPR, clinical pregnancy rate.

Table 4. Live birth rate by the nature of embryo transfer

\begin{tabular}{lrrrrr}
\hline \multirow{2}{*}{ Variable } & \multicolumn{3}{c}{ Live birth delivery } & \multicolumn{1}{c}{ Total } \\
\cline { 2 - 4 } & Singleton & \multicolumn{1}{c}{ Twin } & Triplet & Quadruplet or more \\
\hline In vitro fertilization & $1,024(76.7)$ & $303(22.7)$ & $8(0.6)$ & $0(0.0)$ & $1,335(100.0)$ \\
Intracytoplasmic sperm injection & $1,472(73.3)$ & $521(25.9)$ & $15(0.7)$ & $1(0.0)$ & $2,009(100.0)$ \\
Thawing embryo transfer & $735(73.4)$ & $261(26.1)$ & $5(0.5)$ & $0(0.0)$ & $1,001(100.0)$ \\
Total & $3,231(74.4)$ & $1,085(25.0)$ & $28(0.6)$ & $1(0.0)$ & $4,345(100.0)$ \\
\hline
\end{tabular}

delivery, a total of 4,345 patients were confirmed to have live births. However, several clinics did not report the number of live births or whether they were accomplished by IVF, ICSI, or thawing ET in 2010 (Table 4).

\section{Fresh cycles}

1) Clinical pregnancy by fertilization methods

Of the 20,903 fresh ET cycles, 33.2\% used IVF and 66.8\% used ICSI procedures. Among the embryos that were successfully transferred, the clinical pregnancy rate was $39.3 \%$ and $29.7 \%$ among those who received an IVF and ICSI procedure, respectively (Table 5).

2) Clinical pregnancy by the number of transferred embryos

Of the 21,133 fresh embryo cycles, nearly three-quarters (73.6\%) of these were two (2-ET) or three embryos transfer (3-ET) cycles (2$\mathrm{ET}=36.3 \%$ and $3-\mathrm{ET}=37.3 \%$ ), and $14.0 \%$ were single ETs. Overall, the clinical pregnancy rate per ET was $35.9 \%$ for 2-ET cycles and 32.9\% for 3-ET cycles.

The multiple pregnancy rate per ET was the highest among women who received 4-ET cycles (10.4\%) followed by women who received

Table 5. Clinical pregnancies by fresh ET according to the fertilization method

\begin{tabular}{lccc}
\hline Variable & IVF & ICSI & All \\
\hline ET (\%) & $6,939(33.2)$ & $13,964(66.8)$ & $20,903(100.0)$ \\
Clinical pregnancies & 2,730 & 4,151 & 6,881 \\
CPR per ET (\%) & 39.3 & 29.7 & 32.9 \\
\hline
\end{tabular}

IVF, in vitro fertilization; ICSI, intracytoplasmic sperm injection; ET, embryo transfer; $\mathrm{CPR}$, clinical pregnancy rate.

${ }^{\text {a) }} \mathrm{A} p$-value $<0.05$. 
3-ET cycles (9.5\%) (Table 6).

\section{Thawing cycles}

1) Clinical pregnancy by fertilization methods

Of the 6,922 thawing ET cycles, 36.0\% used ICSI, 19.4\% used halfICSI and $44.6 \%$ used IVF procedures. The clinical pregnancy rate was $39.3 \%$ for the IVF group, $36.4 \%$ for the half-ICSI group, and $29.7 \%$ for the ICSI group (Table 7).

\section{2) Clinical pregnancy by number of embryos transferred}

Of the 6,648 fresh embryo cycles, approximately $68 \%$ were 2-ET or 3 -ET cycles (2-ET $=43.4 \%$ and $3-\mathrm{ET}=25.4 \%)$, and $18.9 \%$ were single ETs. Overall, the clinical pregnancy rate per ET was $38.9 \%$ for 2-ET cycles and $36.7 \%$ for 3 -ET cycles.

The multiple pregnancy rate per ET was the highest among women who received 4-ET cycles (14.7\%) followed by women who received 5-ET cycles (14.5\%) (Table 8).

\section{Other related procedures}

1) Surgical retrieval of sperm and assisted hatching

A total of 617 cases of surgical sperm retrieval were reported to successfully progress to the embryo transfer stage. The overall clinical pregnancy rate per ET was $31.0 \%$.

Of 3,386 cases of assisted hatching that were attempted, 876 cycles were reported to successfully progress to clinical pregnancies. The overall clinical pregnancy rate per ET was $25.9 \%$ (Table 9).

\section{2) Preimplantation genetic diagnosis}

Of 424 cycles of preimplantation genetic diagnosis that had been planned, a total of 85 cases successfully progressed to the clinical pregnancies after ET. In 2010, a total of 1,761 embryos were examined at the preimplantation stage, and 1,111 of these embryos were diagnosed as normal.

3) Oocyte cryopreservation

In 2010, Oocyte cryopreservation was made in 135 cycles. Twenty-

Table 6. Clinical pregnancy by the number of transferred embryos in the fresh cycles

\begin{tabular}{lccccccc}
\hline \multirow{2}{*}{ Outcome } & \multicolumn{5}{c}{ No. of embryos transferred } & \multicolumn{1}{c}{ Total } \\
\cline { 2 - 6 } & One & Two & Three & Four & Five & Six or more \\
\hline ET cycles (\%) & $2,950(14.0)$ & $7,663(36.3)$ & $7,881(37.3)$ & $2,173(10.3)$ & $443(2.1)$ & $23(0.1)$ & $21,133(100.0)$ \\
Clinical pregnancies & 562 & 2,748 & 2,593 & 676 & 103 & 3 & 6,685 \\
CPR per ET (\%) & 19.1 & 35.9 & 32.9 & 31.1 & 23.3 & 13.0 & 31.6 \\
Two or more gestational sac & 4 & 680 & 751 & 227 & 37 & 2 & 1,701 \\
MPR per ET (\%) & 0.1 & 8.9 & 9.5 & 10.4 & 8.4 & 8.7 & 8.0 \\
\hline
\end{tabular}

$\mathrm{ET}$, embryo transfer; CPR, clinical pregnancy rate; MPR, multiple pregnancy rate.

Table 7. Clinical pregnancy by thawing ET according to the fertilization method in the thawing cycles

\begin{tabular}{lcccc}
\hline & & \multicolumn{2}{c}{ Thawing ET } & Partial ICSI \\
\cline { 2 - 4 } Variable & IVF & ICSI & $1,222(19.4)$ & $6,285(100.0)$ \\
ET (\%) & $2,803(44.6)$ & $2,260(36.0)$ & 445 & 2,147 \\
Clinical pregnancies & 955 & 747 & 36.4 & 34.2 \\
CPR per ET (\%) & 39.3 & 29.7 & & 3 \\
\hline
\end{tabular}

$\mathrm{ET}$, embryo transfer; IVF, in vitro fertilization; ICSI, intracytoplasmic sperm injection; CPR, clinical pregnancy rate.

Table 8. Clinical pregnancy by the number of transferred embryos undergoing thawing ET

\begin{tabular}{lccccccc}
\hline \multirow{2}{*}{ Outcome } & \multicolumn{5}{c}{ No. of thawing embryos transferred } & Total \\
\cline { 2 - 6 } & One & Two & Three & Four & Five & Six or more \\
\hline ET cycles (\%) & $1,255(18.9)$ & $2,882(43.4)$ & $1,690(25.4)$ & $668(10.0)$ & $138(2.1)$ & $15(0.2)$ & $6,648(100.0)$ \\
Clinical pregnancies & 326 & 1,121 & 621 & 246 & 40 & 2 & 2,356 \\
CPR per ET (\%) & 26.0 & 38.9 & 36.7 & 36.8 & 29.0 & 13.3 & 35.4 \\
Two or more G-sac & 8 & 308 & 216 & 98 & 20 & 651 \\
MPR per ET (\%) & 0.6 & 10.7 & 12.8 & 14.7 & 14.5 & 6.7 & 9.8 \\
\hline
\end{tabular}

Data on the number of thawing embryos transferred was missing in 297 patients.

$\mathrm{ET}$, embryo transfer; CPR, clinical pregnancy rate; MPR, multiple pregnancy rate. 
Table 9. Outcomes by the methods of surgical retrieval of sperm and assisted hatching

\begin{tabular}{lccccc}
\hline Variable & OPU cycle & Transfer cycles & Clinical pregnancies & Live birth & CPR per ET (\%) \\
\hline PESA & 46 & 42 & 10 & 4 & 23.8 \\
TESE & 702 & 542 & 174 & 75 & 32.1 \\
MESA & 35 & 33 & 7 & 2 & 21.2 \\
Total & 783 & 617 & 191 & 101 & 31.0 \\
Assisted hatching & 3,506 & 3,386 & 876 & 548 & 25.9 \\
\hline
\end{tabular}

OPU, oocyte pick-up; PESA, percutaneous sperm aspiration; TESE, testicular sperm extraction; MESA, microepididymal sperm aspiration; ET, embryo transfer; CPR, clinical pregnancy rate.

eight cycles (17 cycles for medical causes and 11 cycles for social causes) were reported to be planned for fertility preservation.

\section{4) In vitro maturation}

There were 3,012 in vitro maturation cycles reported in 2010. Of these, 835 had embryos transferred, resulting in 141 clinical pregnancies and 90 live births. Overall, the clinical pregnancy rate per ET was $16.9 \%$.

\section{5) Oocyte donation}

In 2010, oocyte donation was performed in 213 cycles. Of these, 194 had embryos transferred, resulting in 101 clinical pregnancies and 31 live births.

\section{6) Selective fetal reductions}

In 2010, a total of 541 selective fetal reductions were performed to decrease the fetal numbers. The vast majority of selective fetal reductions were performed in twin pregnancies (64.1\%), which were the most common form of multiple pregnancies.

\section{Discussion}

Since the first report was issued at 1995, the number of ART procedures and the number of fertility clinics have increased in Korea. In addition, the clinical pregnancy rate per ET in fresh IVF cycles slightly increased from 2009 (30.9\%) to 2010 (32.9\%).

Seventy-eight of the clinics, which submitted their data to KSAR, reported that 128,863 embryos had been cryopreserved and stored in each clinic as of January 1, 2013.

This survey suggests that the age of the women and the number of embryos transferred play an important role in the success of ART treatments. However, the findings in this report are subject to some serious limitations. First, the data submitted were reported by each ART clinic voluntarily and were not validated by any third parties. Second, data about patient demographics, medical histories, and infertility diagnoses were scare. Third, these data were not linked to the individual patients in whom the procedures were performed.
Despite the aforementioned limitations, KSAR is now attempting to extend the boundaries of this reporting system to data related to other infertile procedures including intrauterine insemination, the miscarriage rate, preimplantation genetic screening, and gestational surrogacy. Furthermore, KSAR is focusing on long-term outcomes of ART therapy such as the congenital anomalies, gestational age at delivery, and birth-weight through their homepage (www.ivfkorea. or.kr), which can be used to locate the Korean or English name of all infertility clinics that were involved in the present survey. Such information will aid in expanding the continuity between infertile couples and healthcare providers. Showing the general success rate of ART such as through the clinical pregnancy rate per cycle in the fresh ART cycle, clinical pregnancy rate per cycle in the thawing cycle, and clinical pregnancy rate in the intrauterine insemination cycle may reassure infertile couples who are seeking options for a future pregnancy. Previous reports have suggested making ART clinics accountable for their treatment success by allowing their statistics to be publically available, as was done with the enactment of the Fertility Clinic Success Rate and Certification Act of 1992 in the U.S and the Swedish National Quality Register for Assisted Reproduction. In Korea, the National Supporting Program for Infertility fueled these critical changes at the government level. Although these data might be seen as a resource to compare success rates among fertility clinics and help prospective parents choose an ART center, these data cannot be used for those purposes for several reasons. First, data on the success rate of ART therapy unfortunately takes several years to be assessed. For example, the present report used data from 2010 that became available in 2014. Thus, these data do not reflect the current status of each clinic. Even though an online registration system is expected to be implemented soon in Korea, we expect that there will still be a long interval between data collection and availability. Unless a strong initiative and financial support becomes available, we expect this interval to be decreased to two years. Moreover, if success rates are published and used by patients to select fertility clinics, these ART centers may increase their success rate by moving patients to the more quick IVF therapy instead of using the appropriate practice techniques [1]. 
Through this survey of voluntarily submitted data from 2010, we reported on the national trends at these centers in Korea. We hope that this information will lessen the informational gap between infertile couples and ART clinics and serve as a reference for quality control and the self-promotion of ART centers in Korea.

\section{Conflict of interest}

No potential conflict of interest relevant to this article was reported.

\section{Acknowledgments}

We are deeply grateful to those who have contributed to the development of this report and helped collect the 2010 ART data. We believe their assistance in making this report will be greatly appreciated by future patients who consider undergoing an ART procedure (Supplement 1).

\section{Supplementary materials}

Supplement 1. Seventy-six in vitro fertilization centers participated in 2010 survey.

Supplemental data can be found at: http://ecerm.org/src/sm/cerm42-8-s001.pdf

Supplement 2. Registration form used in 2010 survey.

Supplemental data can be found at: http://ecerm.org/src/sm/cerm42-8-s002.pdf

\section{Reference}

1. Bayer SR, Michael MA, Alan SP. The Boston IVF handbook of infertility: a practical guide for practitioners who care for infertile couples. Abingdon: Informa Healthcare; 2007. 